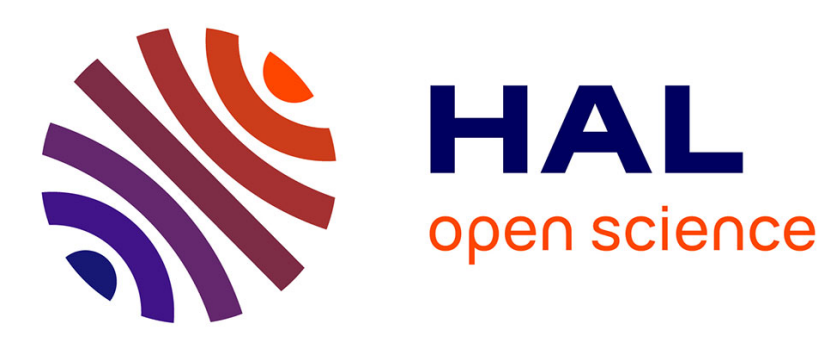

\title{
Pseudodifferential adiabatic mode parabolic equations in curvilinear coordinates and their numerical solution
}

\author{
Pavel S Petrov, Xavier Antoine
}

\section{To cite this version:}

Pavel S Petrov, Xavier Antoine. Pseudodifferential adiabatic mode parabolic equations in curvilinear coordinates and their numerical solution. Journal of Computational Physics, 2020, 410, pp.109392. 10.1016/j.jcp.2020.109392 . hal-02342637

\section{HAL Id: hal-02342637 \\ https://hal.science/hal-02342637}

Submitted on 1 Nov 2019

HAL is a multi-disciplinary open access archive for the deposit and dissemination of scientific research documents, whether they are published or not. The documents may come from teaching and research institutions in France or abroad, or from public or private research centers.
L'archive ouverte pluridisciplinaire HAL, est destinée au dépôt et à la diffusion de documents scientifiques de niveau recherche, publiés ou non, émanant des établissements d'enseignement et de recherche français ou étrangers, des laboratoires publics ou privés. 


\title{
Pseudodifferential adiabatic mode parabolic equations in curvilinear coordinates and their numerical solution
}

\author{
P.S. Petrov ${ }^{\mathrm{a}, *}, \mathrm{X}$. Antoine $^{\mathrm{b}}$ \\ ${ }^{a}$ Il'ichev Pacific oceanological institute, 43 Baltiyskay str., Vladivostok, 690041, Russia \\ ${ }^{b}$ Institut Elie Cartan de Lorraine, Université de Lorraine, UMR 7502, Inria \\ Nancy-Grand Est, F-54506 Vandoeuvre-lès-Nancy Cedex, France
}

\begin{abstract}
Pseudodifferential mode parabolic equations in curvilinear coordinates are derived from the horizontal refraction equations. An energy-conserving splitstep Padé method for their numerical solution is developed. Several examples are considered including the propagation of sound in a perfect wedge, a whispering gallery formation near circular isobath, and the breaking of the whispering gallery waveguide in the vicinity of an inflection point of the isobath.
\end{abstract}

Keywords: parabolic equation method, multiple-scale method, curvilinear coordinates, computational ocean acoustics

$P A C S:$ 43.30.+m,

$P A C S:$ 43.20.Bi

\section{Introduction}

The Mode Parabolic Equations (MPEs) approach is a promising tool for the simulation of sound propagation in shallow-water waveguides. MPEs in ocean acoustics were first derived independently by Collins in [1] and Trofimov in [2] (see also [3]). In their pioneering works, mode coupling was neglected, and the derived equations were valid under the adiabatic assumption. Later on, the theory of narrow-angle MPEs was also generalized to the case of

\footnotetext{
*Corresponding author: P.S. Petrov

Email addresses: petrov@poi.dvo.ru (P.S. Petrov), xavier.antoine@univ-lorraine.fr (X. Antoine)
} 
resonant mode coupling $[2,4,5]$. In many important cases, adiabatic narrowangle MPEs even admit analytical solutions based on group-theoretical techniques $[6,7]$. Recently, it was shown that in waveguides where the horizontal refraction is relatively strong the aperture of narrow-angle MPEs is insufficient to take all horizontal rays into account [4]. In such cases, Wide-Angle MPEs (WAMPEs) must be used to lead to an accurate simulation of the three-dimensional propagation of sound.

In many physical problems, some large portions of the acoustical energy emitted by a source in a shallow sea can be trapped in certain parts of the computational domain. For example, in some recent works, it was shown that, under certain conditions, whispering gallery waves localized in the vicinity of a curvilinear isobath can be excited in a shallow-water waveguide with bowl-like bottom [8] (e.g. in a bay or a lagoon). It is also known that the sound field can be focused by an underwater canyon or trapped between circular fronts of internal waves [9]. For such cases, it is natural to derive and solve mode parabolic equations in curvilinear coordinates.

In the present study, we combine the aforementioned motivations and derive PseudoDifferential [10, 11] MPEs (PDMPEs) in orthogonal curvilinear coordinates. These equations have an arbitrarily wide aperture in the horizontal plane. We also propose a numerical method for solving PDMPEs on unbounded domains in the horizontal plane. This requires an artificial truncation of the computational domain by using Perfectly Matched Layers (PMLs). The numerical solution consists in a marching scheme, where a propagator associated with the PDMPE is evaluated at each step. The propagator is in turn a pseudodifferential operator that is replaced in practice by its Padé approximation. This technique was developed for similar equations complemented by periodicity condition in [12] in the context of a scattering problem.

The plan of the paper is the following. In Section 2, we introduce a modal representation of an acoustical field lying in a 3D shallow-water waveguide and provide the horizontal refraction equation. The pseudodifferential mode parabolic equation in curvilinear coordinates is given in Section 3. Its numerical solution, based on a marching algorithm, is detailed in Section 4. Three numerical applications are developed in Section 5. Finally, we conclude in Section 6 . 


\section{Modal representation of an acoustical field in a 3D shallow-water waveguide and the horizontal refraction equation}

In this Section, we recall some key notions and equations related to adiabatic theory of sound propagation in 3D shallow-water waveguides. The physical conditions for the use of adiabatic approximation, as well as associated shortcomings and implications, are considered in many studies, e.g. in $[13,14]$. Therefore, we do not discuss them here.

MPEs are usually derived [1, 2] from the three-dimensional Helmholtz equation for a sound field in shallow-water produced by a monotone point source located at $x=x_{s}, y=y_{s}, z=z_{s}$, i.e.

$$
\frac{\partial^{2} P}{\partial x^{2}}+\frac{\partial^{2} P}{\partial y^{2}}+\frac{\partial^{2} P}{\partial z^{2}}+\frac{\omega^{2}}{c^{2}} P=-\delta\left(x-x_{s}, y_{s}, z-z_{s}\right),
$$

where $\omega=2 \pi f$ is the cyclic frequency and $c$ denotes the sound speed. In order to properly formulate the sound propagation problem, some interface and boundary conditions are required to be set up in addition to the Helmholtz equation (in particular, in shallow-water the continuity conditions for sound pressure and particle velocity at the water-bottom interface are of great importance). The complete formulation can be found in many textbooks and articles $[3,6,13]$.

The solution of (2.1) can be represented in the form of the modal expansion

$$
P(x, y, z)=\sum_{j=1}^{N_{m}} \mathcal{A}_{j}(x, y) \phi_{j}(z, x, y),
$$

where $\mathcal{A}_{j}(x, y)$ are modal amplitudes, and $\phi_{j}(z, x, y)$ are (local) mode functions computed for the media parameters (including the sound speed profile) at the point $(x, y)$. Mode functions can be found from acoustical spectral problems $[6,13,15]$. Numerous methods for its solution have been developed in the literature [13].

Under the assumption on the adiabaticity of the sound propagation, it can be shown that modal amplitudes satisfy the so-called Horizontal Refraction Equations (HREs) [1, 13]

$$
\frac{\partial^{2} \mathcal{A}_{j}}{\partial x^{2}}+\frac{\partial^{2} \mathcal{A}_{j}}{\partial y^{2}}+k_{j}^{2} \mathcal{A}_{j}=-\delta(x) \delta(y) \frac{\phi_{j}\left(z_{s}\right)}{\rho_{w}},
$$

where $k_{j}=k_{j}(x, y)$ is the horizontal wavenumber corresponding to the eigenfunction $\phi_{j}(z, x, y)$ and $\rho_{w}$ is the density in the water column. 


\section{Pseudodifferential mode parabolic equation in curvilinear coor- dinates}

In many cases, it is natural to derive and solve MPEs in curvilinear coordinates. For example, in [8], the authors considered whispering gallery waves formed in the vicinity of a curvilinear isobath due to the horizontal refraction of acoustical waves by the sloping bottom. Such waves can be accurately described in an orthogonal coordinate system where one of the coordinate lines coincides with the isobath (e.g., if the isobath is circular then the polar coordinate system should be used). As mentioned in the introduction, there also exist other propagation problems of shallow-water acoustics where it is natural to use curvilinear coordinates (e.g., the ducting of sound between the curved fronts of internal waves). For the rest of this study, we will mostly associate our coordinates with curved isobaths to have a concrete example where our results can be applied. However, it should be clear for the reader that in the general case one family of the curvilinear coordinate lines must coincide with level sets of the function $k_{j}=k_{j}(x, y)$. If sound speed in the water is assumed to be constant, these level sets coincide with isobaths. Furthermore, our approach is also applicable to the solution of PDMPEs in Cartesian coordinate systems that are sufficient to handle the majority of sound propagation problems of shallow-water acoustics.

Assume that the source and the receiver are located on the same circular isobath where whispering gallery waves are excited. Our ultimate goal is to simulate both these waves creeping along an arc of the circle and direct-path propagation from the source to the receiver along the circle's chord. In order to tackle this problem, as well as more general cases where isobaths have more complicated forms, we need a WAMPE with an aperture of $\pm 45^{\circ}$ that is solved along the isobath.

Let us assume that the isobath that supports whispering gallery waves in its vicinity is given by a parametric equation

$$
x=X(s), y=Y(s),
$$

where $s$ is the arclength. We start by introducing an orthogonal coordinate system $(s, \xi)$ in its vicinity, where the $\xi$-axis is aligned along the depth gradi-

ent $\left(\frac{\partial h}{\partial x}, \frac{\partial h}{\partial y}\right)$ and has the opposite direction (clearly, the $\xi$-axis has the same direction as the unit normal vector $\mathbf{n}=\left(Y^{\prime}(s),-X^{\prime}(s)\right)$ to the isobath (3.1). 
Any point $(s, \xi)$ in the vicinity of the isobath has the Cartesian coordinates

$$
\left\{\begin{array}{l}
x=X(s)+n Y^{\prime}(s) \\
y=Y(s)-n X^{\prime}(s)
\end{array}\right.
$$

In the curvilinear coordinates $(s, \xi)$, the $\operatorname{HRE}(2.3)$ writes as

$$
\frac{1}{\gamma} \frac{\partial}{\partial \xi}\left(\gamma \frac{\partial \mathcal{A}_{j}}{\partial \xi}\right)+\frac{1}{\gamma} \frac{\partial}{\partial s}\left(\frac{1}{\gamma} \frac{\partial \mathcal{A}_{j}}{\partial s}\right)+k_{j}^{2} \mathcal{A}_{j}=0
$$

where we omitted the source term on the right-hand side (this it typical for the derivation of PEs of various types). In (3.3), the variable coefficient $\gamma=\gamma(s, \xi)=1+\xi \varkappa(s)$ is associated with the geometry of isobath, and $\varkappa(s)$ is the curvature of the latter. It can be computed as the length of the derivative of the unit tangent vector $\mathbf{t}=\mathbf{t}(s)$ to the isobath

$$
\varkappa(s)=\left|\frac{d \mathbf{t}}{d s}\right|=\sqrt{\left(\frac{d^{2} X}{d s^{2}}\right)^{2}+\left(\frac{d^{2} Y}{d s^{2}}\right)^{2}} .
$$

Derivation of PDMPEs corresponding to (3.3) can be greatly simplified by getting rid of the divergence form with respect to the arclength variable $s$. This can be accomplished by the substitution $\mathcal{A}_{j}(s, \xi)=\sqrt{\gamma} v(s, \xi)^{1}$. The equation for $v=v(s, \xi)$ has the following form

$$
v_{s s}+\left(\gamma^{2} v_{\xi}\right)_{\xi}+\left[\gamma^{2} k^{2}+\frac{1}{4}\left(\gamma_{\xi}\right)^{2}+\frac{1}{2} \frac{\gamma_{s s}}{\gamma}-\frac{3}{4} \frac{\left(\gamma_{s}\right)^{2}}{\gamma^{2}}\right] v=0
$$

It can be also written in the operator form

$$
v_{s s}=-\hat{L}(s) v
$$

where

$$
\hat{L}(s)=\frac{\partial}{\partial \xi}\left(\gamma^{2}\right) \frac{\partial}{\partial \xi}+\left[\gamma^{2} k^{2}+\frac{1}{4}\left(\gamma_{\xi}\right)^{2}+\frac{1}{2} \frac{\gamma_{s s}}{\gamma}-\frac{3}{4} \frac{\left(\gamma_{s}\right)^{2}}{\gamma^{2}}\right]
$$

is the transverse operator with respect to the propagation direction along the isobath.

\footnotetext{
${ }^{1}$ Hereafter, we omit the subscript $j$ indicating the mode number, and denote the partial derivatives by the subscripts $s, \xi$, etc.
} 
From (3.6), a pseudodifferential PE (PDPE) can be written in the most obvious way as

$$
v_{s}=\mathrm{i} \sqrt{\hat{L}(s)} v
$$

However, from general considerations, it is known that a better option is offered by an energy-conserving counterpart $[12,16,17,18,19]$ of the latter equation. It can be obtained by introducing a new unknown $\varphi(s, \xi)=$ $\sqrt[4]{L(s)} v(s, \xi)$, and it can be easily shown that a PDPE for $\varphi$ has the same form

$$
\varphi_{s}=\mathrm{i} \sqrt{\hat{L}(s)} \varphi
$$

Eq. (3.7) is called hereafter PseudoDifferential MPE (PDMPE). This nonlocal equation can be solved by using some Padé approximations for the square-root operator $\sqrt{\hat{L}(s)}$.

The general recipe for the computation of an acoustical field can be outlined as follows:

1. Compute the modal wavenumbers $k_{j}(x, y)$ by solving acoustical spectral problems for all $(x, y)$ (in practice, this can be greatly simplified by using mode perturbation theory).

2. Identify some suitable curvilinear coordinates $(s, \xi)$ in such a way that the coordinate lines $\xi=$ const coincide with the level sets of the function $k_{j}(x, y)$ (in many cases, it can be Cartesian coordinate system $(x, y)$ chosen in a standard way with $x$-axis connecting the source and the receiver).

3. For each mode number $j$, solve Eq. (3.7) and compute $v(s, \xi)$ by the formula $v(s, \xi):=(\hat{L}(s))^{-1 / 4} \varphi(s, \xi)$.

4. Evaluate $\mathcal{A}_{j}(x, y)$ by transforming the function $\mathcal{A}_{j}(s, \xi)=\gamma v(s, \xi)$ back to the Cartesian coordinates $(x, y)$.

5. Use the modal expansion (2.2) to compute the acoustical pressure $P(x, y, z)$.

\section{Numerical solution of the PDMPE: a marching algorithm}

Following [12], we introduce a uniform grid $\left\{s_{n}\right\}$ with step size $h$ (i.e., such that $\left.s_{n+1}=s_{n}+h\right)$. Equation (3.7) can be formally solved by a marching scheme $[20]$

$$
\varphi_{n+1}=\exp \left(\mathrm{i} k_{0} h \sqrt{1+\hat{X}}\right) \varphi_{n}:=P \varphi_{n}
$$


where $k_{0}$ is the reference wavenumber, $L=k_{0}^{2}(1+\hat{X})$, and the operator $X$ is evaluated at the mid-point $s_{n+1 / 2}=s_{n}+h / 2$. The exponential operator $P$ is called the one-way propagator corresponding to (3.7). Note that the respective propagators for narrow-angle MPE in many cases can be evaluated globally (for arbitrarily large steps) by various operator disentanglement analytical methods (even in the case of variable coefficients of special form, see $[7,21])$. By contrast, the more complicated case of (3.7) can be handled only numerically.

\subsection{Approximation of the propagator $P$ in Eq. (4.1)}

In this study we also use the same $\theta$-approximant for the propagator $P$ as described in [20]

$$
\varphi_{n+1}=P \varphi_{n} \approx \mathrm{e}^{\mathrm{i} k_{0} h}\left(d_{0}+\sum_{k=1}^{p} \frac{d_{k}}{1+b_{k} \hat{X}}\right) \varphi_{n}=\mathrm{e}^{\mathrm{i} k_{0} h}\left(d_{0} \varphi_{n}+\sum_{k=1}^{p} d_{k} w_{n, k}\right),
$$

where $w_{n, k}$ is obtained by solving the following auxiliary PDE

$$
\left(1+b_{k} \hat{X}\right) w_{n, k}=\varphi_{n} .
$$

\subsection{Domain truncation and the PMLs}

In the majority of practical problems of underwater acoustics, there exist areas, where the sound field has to be computed, that have no physical boundaries in horizontal directions. Thus, the problem of artificial domain truncations naturally arises. It can be solved by introducing a fictitious boundary at which either an artificial (or absorbing) boundary condition $[22,23]$ is set up or a Perfectly Matched Layer (PML) [23, 24, 25] is attached to the computational domain. In this study, we choose the latter approach.

Assume that the solution of Eq. (3.7) is sought on the domain $(\xi, s) \in$ $\Omega=[-d, d] \times\left[0, s_{\max }\right]$ (a curvilinear stripe of width $2 d$ containing the given isobath (3.1)). We increase the width of the stripe by $\epsilon$ from each side and consider an initial-value problem for Eq. (3.7) on the extended domain $\bar{\Omega}=[-d-\epsilon, d+\epsilon] \times\left[0, s_{\max }\right]$, where the operator $\hat{L}$ is replaced by its PML version

$$
\hat{L}_{P M L}=\frac{1}{1+\mathrm{i} \sigma(\xi)} \frac{\partial}{\partial \xi} \frac{\gamma^{2}}{1+\mathrm{i} \sigma(\xi)} \frac{\partial}{\partial \xi}+\left[\gamma^{2} k^{2}+\frac{1}{4}\left(\gamma_{\xi}\right)^{2}+\frac{1}{2} \frac{\gamma_{s s}}{\gamma}-\frac{3}{4} \frac{\left(\gamma_{s}\right)^{2}}{\gamma^{2}}\right],
$$

for a smooth function $\sigma(\xi)$ which is increasing in $\xi$ on $[d, d+\epsilon]$, decreasing on $[d-\epsilon, d]$, and such that $\sigma(\xi)=0$ for $\xi \in[-d, d]$. Thus, the operator $\hat{L}_{P M L}$ 
coincides with $\hat{L}$ on $[-d, d]$, and outside this interval the derivatives $\frac{\partial}{\partial \xi}$ in $\hat{L}$ are replaced by $\frac{1}{1+\mathrm{i} \sigma(\xi)} \frac{\partial}{\partial \xi}$.

Our numerical scheme implements the solution of an initial-boundary value problem for Eq. (3.7) on the domain $\bar{\Omega}$ with homogeneous Dirichlet boundary conditions of the form $\left.\varphi\right|_{\xi= \pm d \pm \epsilon}=0$ at the boundaries $\xi= \pm d \pm \epsilon$ of the PMLs. Inside the domain $\Omega$, this solution accurately approximates the solution of Eq. (3.7) on the unbounded domain $-\infty<\xi<\infty, s \geq 0$ provided that $\sigma(\xi)$ is chosen in a suitable way. In the paper, we fix $\sigma$ to $\sigma(\xi)=\sigma_{0}(\xi-d)^{3} / \epsilon^{3}$ (in the examples presented below $\sigma_{0}=5, \epsilon=300 \mathrm{~m}$ ).

\subsection{Discretization of the operator $X$}

In order to solve the operator equations (4.3), we must discretize the operator $X$. It can be done by many techniques, but for the purposes of this study it is sufficient to use a standard second-order finite-difference method on a uniform grid $\left\{\xi_{\ell}\right\}$, where $\xi_{\ell+1}=\xi_{\ell}+\tau$. Thus, each operator equation (4.3) reduces to a tridiagonal system of linear algebraic equations.

\subsection{Initial conditions for PDMPE}

In computational problems of ocean acoustics, initial conditions for PEs (usually called PE starters) are chosen in such a way that the solution of the respective Initial-Boundary Value Problem (IBVP) approximates the solution of the Helmholtz equation (2.1) with a point source on the right-hand side. Many starters were developed for parabolic equations of various types (see e.g. [13]) in past decades. In our work, we use the so-called Greene's starter

$$
\mathcal{A}_{j}(\xi, 0)=\frac{\phi_{j}\left(z_{s}\right)}{2 \sqrt{\pi}}\left(1.4467-0.8402 k_{j, 0}^{2} \xi^{2}\right) e^{-\frac{k_{j, 0}^{2} \xi^{2}}{1.5256}} .
$$

Let us remark that this starter is probably non-optimal for PDMPE, as it was designed for a rational-linear PE. A development of the starter consistent with (3.7) will be the subject of a future work.

\section{Examples}

\subsection{Sound propagation in a coastal wedge}

In the first example, we consider the sound propagation in a wedge-shaped waveguide (see Fig. 1) that is a simplified model of a near-coastal area of a shallow sea. This is a standard test scenario used for validation of various 
3D computational models in ocean acoustics. Although for many approaches the case of wedge with penetrable bottom is particularly challenging, this is not the case of MPEs. Indeed, difficulties associated with bottom properties are all confined to the solution of acoustical spectral problem (i.e., to the procedure of computation of $k_{j}$ and $\left.\phi_{j}(z, \xi, s)\right)$. On the other hand, perfectly reflecting bottom yields stronger manifestation of the horizontal refraction effect, and therefore is more suitable for validation of MPE-based models.

Clearly, in this example, it is natural to use rectilinear Cartesian coordinates, where $\kappa=0$ and $\gamma=1$. However, to the best of our knowledge, the wedge problem has never been solved until now using wide-angle MPEs or PDMPEs. For our example, we take the bottom slope angle $\alpha=1^{\circ}$, the sound frequency $f=100 \mathrm{~Hz}$ and the water depth at the source location $h_{0}=58 \mathrm{~m}$. The sound speed in the water is $1500 \mathrm{~m} / \mathrm{s}$.

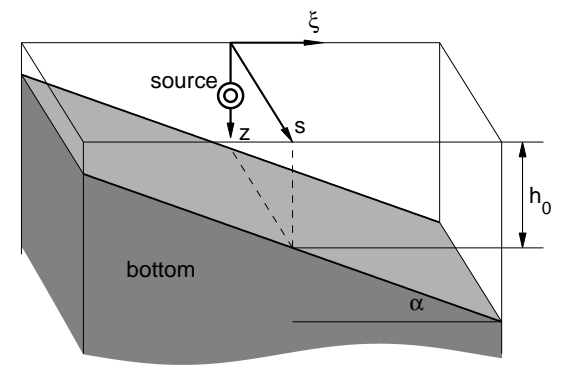

Figure 1: Coastal wedge: a simplified model of a shallow sea with sloping bottom. The variable $s$ is the distance from the source in the direction along the isobath, $\xi$ is aligned along the depth gradient.

The IBVP for Eq. (3.7) with initial conditions (4.4) is solved on the domain $\Omega=\{(\xi, s) /|\xi| \leq 3 \mathrm{~km}, 0 \leq s \leq 14 \mathrm{~km}\}$. At the boundaries $\xi=$ $\pm 3 \mathrm{~km}$, PMLs with thickness $\epsilon=300 \mathrm{~m}$ are added to the computational domain. The solution is obtained on a uniform grid with step size $h=2 \mathrm{~m}$ (the discretization step $\Delta \xi$ for the operator $X$ is $0.5 \mathrm{~m}$ ).

The contour plot of the computed solution is presented in logarithmic units in Fig. 2 (left panel). It is validated by a direct comparison with the solution provided by the method of source images [26] (see right panel of Fig. 2). It can be concluded from Fig. 2 that the two solutions are almost identical, although some small discrepancies are visible near the source (no- 

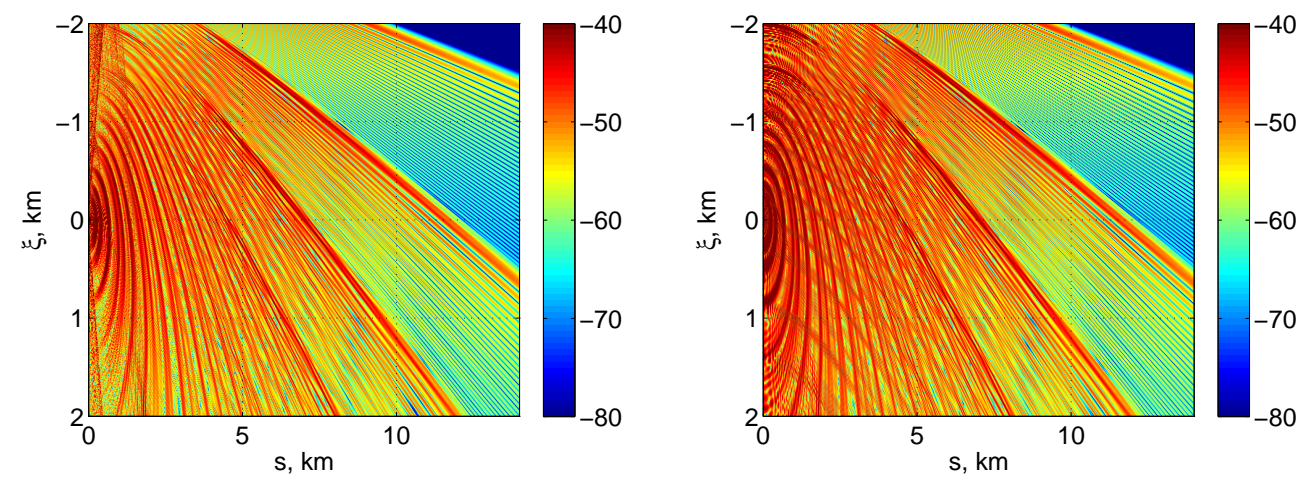

Figure 2: Acoustical field in a wedge-shaped waveguide computed by the PDMPE (3.7) (left) and using the source images method [26] (right). The solutions have noticeable discrepancies near the source (especially in the area of PDMPE sidelobes) but perfectly coincide in the far-field.

tice the sidelobes produced by PDMPE). For a more detailed comparison, we plotted the two solutions at $\xi=0$ in Fig. 3, and the agreement appears to be nearly perfect.

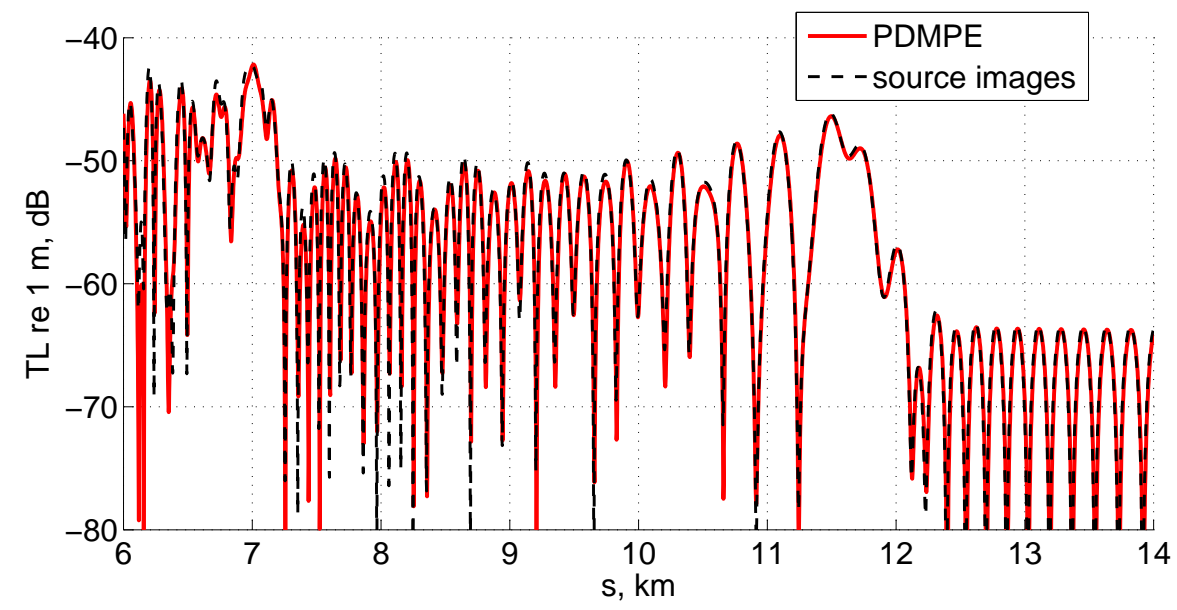

Figure 3: Comparison of the solutions obtained. 


\subsection{Whispering gallery waves in the vicinity of a circular isobath (constant curvature case)}

In the second example, we consider the sound propagation in a shallowwater with circular isobaths depicted in Fig. 4 (depth gradient must have the same direction as the normal vector to the isobath). As shown in [8], under certain conditions, horizontal refraction in such a waveguide can result in the formation of Whispering Gallery (WG) waves [27] localized in the vicinity of an isobath. The properties of such waves and their modal structure was studied in [8] using a WKBJ method. In this work, we present the results of the direct computation of the acoustical field produced by a point source in a waveguide of this type by the numerical solution of the PDMPE (3.7).

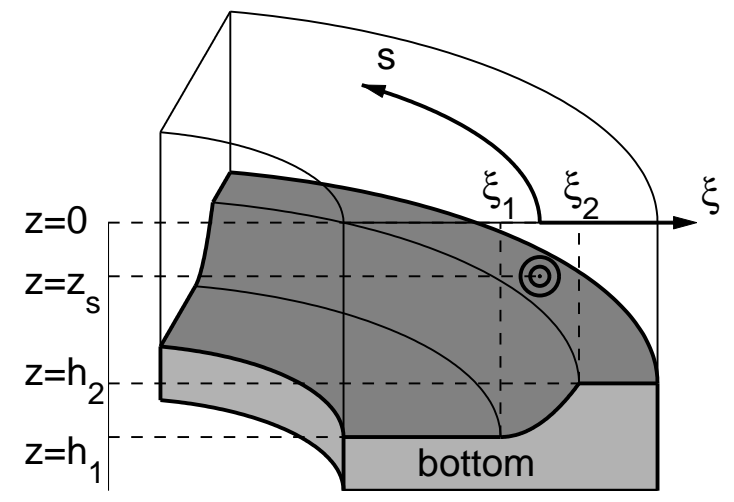

Figure 4: An area of a shallow sea with circular isobaths. The variable $s$ is the distance from the source along the circular isobath, $\xi$ is aligned along the depth gradient. The depth is constant for $\xi>\xi_{2}$ and for $\xi<\xi_{1}$

Let us introduce the polar coordinate system $(r, \theta)$ in the horizontal plane in such a way that its center coincides with the curvature center of the isobaths. Let us assume that the bathymetry is described by a linear function $h(r)$ on the interval $\left[r_{1}, r_{2}\right]$ (the same as in [8]), i.e.

$$
h(r)=h(r)=\left\{\begin{array}{l}
h_{1} \text { if } r \leq r_{2}, \\
h_{1}+\frac{h_{2}-h_{1}}{r_{2}-r_{1}}\left(r-r_{1}\right) \text { if } r \in\left[r_{1}, r_{2}\right], \\
h_{2} \text { if } r \geq r_{2} .
\end{array}\right.
$$


The parameters are chosen as follows: $r_{1}=5.8 \mathrm{~km}, r_{2}=6 \mathrm{~km}, h_{1}=26 \mathrm{~m}$, $h=h_{2}=24 \mathrm{~m}$. In this example (as well as in the next one), the water column and the bottom are isovelocity layers, where the values of the sound speed are $c_{w}=1500 \mathrm{~m} / \mathrm{s}$ and $c_{b}=2000 \mathrm{~m} / \mathrm{s}$, respectively. The densities in these layers are $\rho_{w}=1 \mathrm{~g} / \mathrm{cm}^{3}, \rho_{b}=1.8 \mathrm{~g} / \mathrm{cm}^{3}$, respectively. We assume that an omnidirectional point source of frequency $f=120 \mathrm{~Hz}$ is located at $z=z_{s}=10 \mathrm{~m}, r=r_{s}=5800 \mathrm{~m}$ and $\theta=\theta_{s}=0$. In this case, it is natural to use curvilinear coordinates as

$$
\xi=r-r_{s}, \quad s=r_{s} \theta
$$

since WG waves are expected to be formed in the vicinity of the isobath $r=r_{s}$ (see Fig. 4).

The considered shallow-water waveguide supports three vertical modes for all values of $r$ and $\theta$. As shown in [8], the WG-waveguide is formed in this case only for the highest-order vertical mode, i.e., for $j=3$. In turn, the mode 3 component of the field $P_{3}(r, \theta, z)=A_{j}(r, \theta) \phi_{j}(z, r, \theta)$ exhibits a modal structure in the horizontal plane due to the formation of WG-modes. The energy transmitted by these modes is localized near the isobath $r=$ $r_{s}$, and does not undergo cylindrical spreading. Thus, in the far-field, the contribution of WG modes becomes dominant as the divergent part of the field fades away.

The method for the computation of the WG-component of $P_{3}(r, \theta, z)$ is described in [8]. It is based on the WKBJ approximation for the angular wavenumbers of WG modes and semi-analytical computation of radial mode functions. The solution magnitude in decibels $20 \log _{10}\left(4 \pi P_{3}\right)$ for our example is shown in Fig. 5 (right panel) at the depth $z=z_{s}$ (the magnitude at $1 \mathrm{~m}$ from the source as the reference level). We also computed $P_{3}\left(r, \theta, z=z_{s}\right)$ by solving PDMPE in the curvilinear coordinates $(s, \theta)$. The result is presented in Fig. 5 (left panel), and the comparison with the WG-component of $P_{3}$ calculated by the method from [8] along the arc $r=r_{s}$ is shown in Fig. 6 .

For a better illustration of this result, we also converted the PDMPE solution to the Cartesian coordinates (see Fig. 7). It can be seen that a certain part of the acoustical energy generated by the source is ducted in the vicinity of the isobath $r=r_{s}$ due to the horizontal refraction over the bottom slope. Although all horizontal rays corresponding to the mode 3 become bent due to this phenomenon, some of them do not get trapped in the WG-waveguide and leave the circle $r=r_{2}$. After the respective energy 

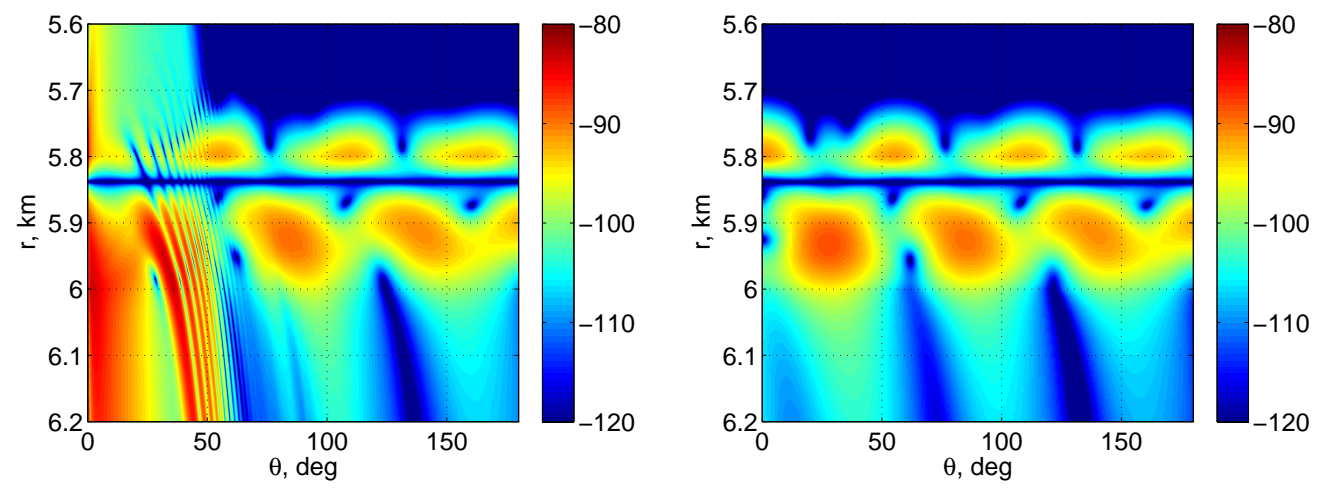

Figure 5: Acoustical field of the third mode $A_{3}(s, \xi)$ in a waveguide with circular isobaths computed by the PDMPE (3.7) (left) and using the WKB approximation for whispering gallery modes (right). The solutions perfectly coincide at long range, where the wave field is dominated by whispering gallery waves.

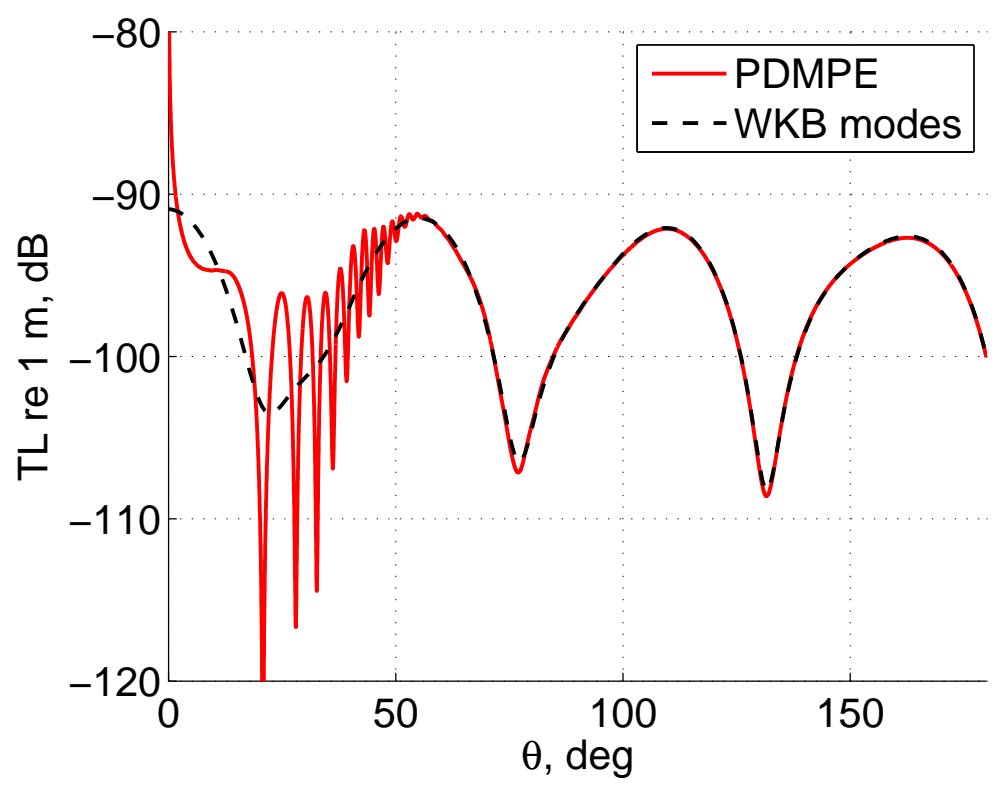

Figure 6: Comparison of the two solutions shown in Fig. 5 along the arc $r=r_{s}$ (i.e., $\xi=0)$.

is radiated into the outer space, the remaining part is formed exclusively by the WG-waves (as shown in Fig. 6).

In this example, we have $\varkappa=\varkappa(s)=1 / r_{s}$. In addition, the comparison in 


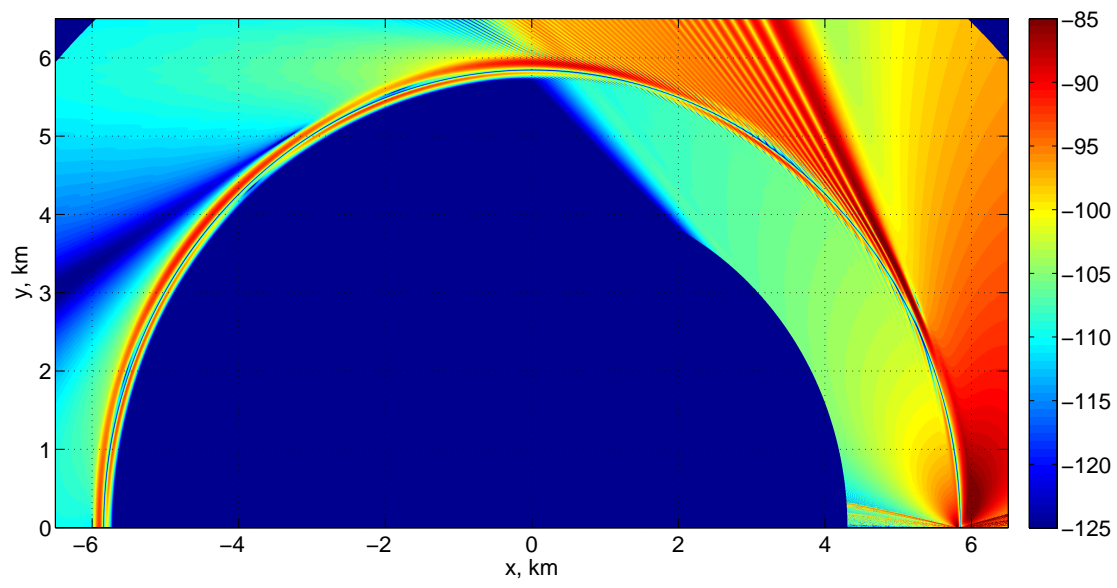

Figure 7: Acoustical field in a an area of a shallow sea with circular isobaths where whispering gallery waves are formed due to horizontal refraction.

Fig. 6 indicates that the PDMPE accurately takes the curvilinear geometry of the domain into account.

\subsection{Dissipation of a WG wave: inflection point of an isobath}

In the final example of our study, we consider a problem for which no analytical solution is available (apparently it cannot be also obtained by the MPE methods in a Cartesian coordinate system). In this case, radiation of the energy ducted in a WG-waveguide into the free-space will take place due to the presence of an inflection point of the isobath. This is an underwater acoustics counterpart of the WG propagation problem where the boundary of the domain has an inflection point first considered by Popov and his coauthors $[28,29]$ (both analytically and numerically). Some asymptotics of the field in shadow zones as well as the behaviour of the solution at infinity was also studied by Babich and Smyshlyaev [30, 31] (see also the recent article [32] and references therein).

It is known that usual whispering gallery waves are formed near the reflective boundary of a convex domain. If at some point the curvature of the boundary changes its sign (i.e., the adjoint part of the domain is no longer convex), then the waveguide for WG waves ceases to exist, and the lion's share of their energy is radiated into the interior of the domain in the form of a searchlight beam. At the same time, minor part of the ducted energy 
penetrates into the shadow zone along the boundary after the inflection point. Clearly, one can expect similar behaviour of the WG waves trapped near a curvilinear isobath in a shallow sea.

Let us assume that a point source of frequency $f=120 \mathrm{~Hz}$ is placed at the isobath defined by the following formula in a Cartesian coordinate system (see also Fig. 8)

$$
\left\{\begin{array}{l}
x=\tilde{X}(t)=x_{0}-t, \\
y=\tilde{Y}(t)=a\left(t-x_{0}\right)^{3}+a x_{0}^{3},
\end{array}\right.
$$

where $x_{0}=4000 \mathrm{~m}$ and $a=10^{-8} \mathrm{~m}^{-2}$ are fixed constants (and $t$ is a nonnatural parametrization of this curve). First, we introduce the arclength parameter $s$ defined by

$$
s=\int_{0}^{t} \sqrt{\tilde{X}^{2}(t)+\tilde{Y}^{2}(t)} d t,
$$

rewrite the curve equations (5.3) as $x=X(s), y=Y(s)$, and compute $\varkappa(s)$ using Eq. (3.4). The curvature radius $1 / \varkappa(s)$ gradually increases from $5.6 \mathrm{~km}$ at $x=x_{0}$ (i.e., from $s=0$ ) to $+\infty$ at $x=0$. At $x=0$, the isobath has an inflection point where the curvature $\varkappa(s)$ changes its sign (see the dashed lines in Fig. 8). Let us introduce the curvilinear coordinates $(s, \xi)$ in the vicinity of this isobath, where $s$ is the aforementioned arclength parameter of the latter, and $\xi$ is the distance to the isobath from a given point in its vicinity. In the vicinity of the isobath, the depth varies only in the transverse direction, i.e.

$$
h(\xi)=\left\{\begin{array}{l}
h_{1} \text { if } \xi \leq \xi_{2}, \\
h_{1}+\frac{h_{2}-h_{1}}{\xi_{2}} \xi \text { if } \xi \in\left[0, \xi_{2}\right], \\
h_{2} \text { if } \xi \geq \xi_{2} .
\end{array}\right.
$$

Obviously, the dependence $h=h(\xi)$ is exactly the same as in the previous example (although in this case it cannot be related to polar coordinates as in Eq. (5.1)), but the geometry of isobaths is more complicated due to the presence of an inflection point at $x=0$. All the other environment parameters (sound speeds, densities, depths, etc) also have the same values as in the previous example.

In this case we also restrict our attention to the mode 3 component of the total field produced by the point source, since for the lower modes horizontal 


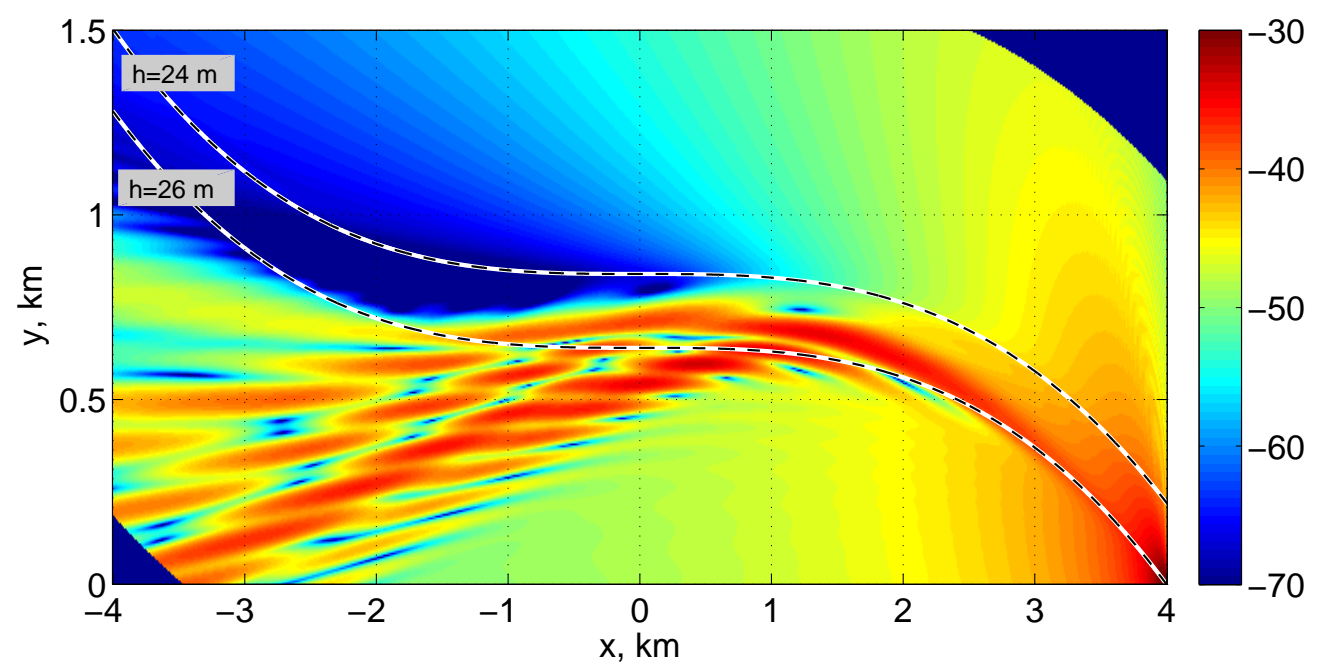

Figure 8: Radiation of the energy trapped in a whispering gallery waveguide into freespace. The isobaths $h=24 \mathrm{~m}$ and $h=26 \mathrm{~m}$ are shown by dashed lines.

refraction does not lead to the formation of a WG waveguide. The contour plot of $P_{3}\left(x, y, z=z_{s}\right)$ in logarithmic units is presented in Fig. 8. The bottom is sloping between the isobaths shown in Fig. 8 by dashed lines and flat outside this area.

From Fig. 8, it is clear that the acoustical energy associated with mode 3 is concentrated in the vicinity of the isobath $h=26 \mathrm{~m}$ for $x>0$ (thus, a WG is formed similarly to the previous example). For $x<0$, i.e. after the inflection point, the WG waves form a searchlight beam and are radiated in the negative direction of the $x$ - and $y$-axes. The diffracted wave further propagating into the shadow zone is also visible.

\section{Conclusion}

In this paper, we derived PDMPEs in orthogonal curvilinear coordinates. The derived equations can be used for the simulation of one-way propagation of sound in shallow-water waveguides with complicated bottom environment inhomogeneities that lead to complicated geometry of horizontal rays and to manifestation of various sound propagation effects. Some of these effects, e.g. whispering gallery formation and breaking, cannot be accurately simulated by using even very wide-angle MPEs in standard Cartesian or polar 
(cylindrical) coordinate systems since the propagation direction can change by $180^{\circ}$ at certain distance from the source. Due to the same reason, such problems also cannot be handled by three-dimensional parabolic equations.

At the same time, the examples in Sections 5.1 and 5.3 cannot be properly handled by narrow-angle MPEs similar to the equations derived in [27] and [29]. Thus, WAMPEs or PDMPEs (similar to the one used in [12]) are required in such cases.

It should be stressed that PDMPEs considered in our work are obtained under the adiabatic assumption and do not take mode coupling effects into account. However, for many practical problems, the horizontal refraction of sound is more important than the mode interaction, especially when the sound localization near an isobath is expected (or, more generally, near the level set of the horizontal wavenumber $\left.k_{j}=k_{j}(x, y)\right)$.

\section{Acknowledgements}

The reported study was accomplished during P.S. Petrov's visit to the Institut Elie Cartan de Lorraine (Université de Lorraine, France) supported by the French government in the framework of the Metchnikov 2019 program. P.S. Petrov was also partly supported by the Russian Foundation for Basic Research (RFBR) under the contracts No. 18-35-20081_mol_a_ved and No. 18-05-00057_a. X. Antoine was supported by the French National Research Agency project NABUCO, grant ANR-17-CE40-0025.

\section{References.}

[1] M. D. Collins, The adiabatic mode parabolic equation, The Journal of the Acoustical Society of America 94 (4) (1993) 2269-2278.

[2] M. Y. Trofimov, Narrow-angle parabolic equations of adiabatic singlemode propagation in a horizontally inhomogeneous shallow sea, Acoustical Physics 45 (1999) 575-580.

[3] P. S. Petrov, M. Y. Trofimov, A. D. Zakharenko, Mode parabolic equations for the modeling of sound propagation in 3d-varying shallow water waveguides, in: Days on Diffraction (DD), 2012, IEEE, 2012, pp. 197202.

[4] M. Y. Trofimov, S. Kozitskiy, A. Zakharenko, A mode parabolic equation method in the case of the resonant mode interaction, Wave Motion 58 (2015) 42-52. 
[5] A. T. Abawi, W. A. Kuperman, M. D. Collins, The coupled mode parabolic equation, The Journal of the Acoustical Society of America 102 (1) (1997) 233-238.

[6] P. S. Petrov, F. Sturm, An explicit analytical solution for sound propagation in a three-dimensional penetrable wedge with small apex angle, The Journal of the Acoustical Society of America 139 (3) (2016) 13431352.

[7] P. S. Petrov, S. V. Prants, T. N. Petrova, Analytical Lie-algebraic solution of a $3 \mathrm{~d}$ sound propagation problem in the ocean, Physics Letters A 381 (2017) 1921-1925.

[8] B. Katsnelson, P. Petrov, Whispering gallery waves localized near circular isobaths in shallow water, The Journal of the Acoustical Society of America 146 (3) (2019) 1343-1352.

[9] J. F. Lynch, Y.-T. Lin, T. F. Duda, A. E. Newhall, Acoustic ducting, reflection, refraction, and dispersion by curved nonlinear internal waves in shallow water, IEEE Journal of Oceanic Engineering 35 (1) (2010) $12-27$.

[10] M. E. Taylor, Pseudodifferential operators, Princeton Univ. Press, 1981.

[11] L. Hörmander, The analysis of linear partial differential operators, Vol. I to IV, Springer-Verlag, 1983-1985.

[12] X. Antoine, Y. Huang, Y. Y. Lu, Computing high-frequency scattered fields by beam propagation methods: A prospective study, Journal of Algorithms \& Computational Technology 4 (2) (2010) 147-166.

[13] F. B. Jensen, M. B. Porter, W. A. Kuperman, H. Schmidt, Computational ocean acoustics. 2nd edition, Springer, 2011.

[14] A. D. Pierce, Extension of the method of normal modes to sound propagation in an almost-stratified medium, The Journal of the Acoustical Society of America 37 (1) (1965) 19-27.

[15] B. Katsnelson, V. Petnikov, J. Lynch, Fundamentals of shallow water acoustics, Springer Science \& Business Media, 2012. 
[16] O. A. Godin, Reciprocity and energy conservation within the parabolic approximation, Wave motion 29 (2) (1999) 175-194.

[17] L. M. Brekhovskikh, O. A. Godin, Wave propagation in a range dependent waveguide, in: Acoustics of Layered Media II, Springer, 1999, pp. 243-360.

[18] Y. Y. Lu, Improving beam propagation method for TM polarization, Optics and Quantum Electronics 35 (4) (2003) 507-519.

[19] X. Antoine, P. Dreyfuss, K. Ramdani, A construction of beam propagation methods for optical waveguides, Communications in Computational Physics 6 (3) (2009) 565-576.

[20] Y. Y. Lu, Some techniques for computing wave propagation in optical waveguides, Communications in Computational Physics 1 (6) (2006) 1056-1075.

[21] P. N. Petrov, P. S. Petrov, Asymptotic solution for the problem of sound propagation in a shallow sea with the bathymetry described by a parametric quadratic function, The Journal of the Acoustical Society of America 146 (3) (2019) 1946-1955.

[22] X. Antoine, A. Arnold, C. Besse, M. Ehrhardt, A. Schädle, A review of transparent and artificial boundary condition techniques for linear and nonlinear Schrödinger equations, Communications in Computational Physics 4 (2008) 729-796.

[23] X. Antoine, E. Lorin, Q. Tang, A friendly review of absorbing boundary conditions and perfectly matched layers for classical and relativistic quantum waves equations, Molecular Physics 115 (15-16) (2017) 18611879.

[24] J.-P. Berenger, A perfectly matched layer for the absorption of electromagnetic waves, J. Comput. Phys. 114 (1994) 185-200.

[25] F. Collino, Perfectly matched absorbing layers for the paraxial equations, INRIA Rapport de recherche no 2964.

[26] J. Tang, P. Petrov, S. Piao, S. Kozitskiy, On the method of source images for the wedge problem solution in ocean acoustics: Some corrections and appendices, Acoustical Physics 64 (2) (2018) 225-236. 
[27] V. M. Babič, V. S. Buldyrev, Asymptotic methods in short-wavelength diffraction theory, Alpha Science International Limited, 2009.

[28] M. Popov, Problem of whispering gallery waves in a neighborhood of a simple zero of the effective curvature of the boundary, Journal of Soviet Mathematics 11 (5) (1979) 791-797.

[29] M. Popov, I. Pshenchik, Numerical solution of the problem on whispering gallery waves in a neighborhood of a simple zero of the effective curvature of the boundary, Journal of Soviet Mathematics 11 (5) (1979) 797-804.

[30] V. Babich, V. Smyshlyaev, Scattering problem for the Schrödinger equation in the case of a potential linear in time and coordinate. I. Asymptotics in the shadow zone, Journal of Soviet Mathematics 32 (2) (1986) 103-112.

[31] V. Babich, V. Smyshlyaev, The scattering problem for the Schrödinger equation with a potential linear in time and in space. II. correctness, smoothness, behavior of the solution at infinity, Journal of Soviet Mathematics 38 (1) (1987) 1562-1576.

[32] D. Hewett, J. Ockendon, V. Smyshlyaev, Contour integral solutions of the parabolic wave equation, Wave Motion 84 (2019) 90 - 109. 